\title{
Age-related difference of substances controlling mitoses in lymph is a new way of drugs design and development
}

\begin{abstract}
Objective: Mitosis is a fundamental age-related process. Mitoses are in different tissues and they are regulated by mediators. Mediators, for example, cytokines, hormones, are used as drugs for cancer, graft-versus-host disease and other diseases. Our goal is to assess available research pertinent to age-related mitoses, cytokines and hormones in lymph (body fluid), in particular, in thoracic duct lymph (TDL) in norm and use them in new drug design and development.
\end{abstract}

Methods: We investigated the data banks of PubMed/Medline, World Cat, Google and Index Copernicus searching for articles published in the past four decades.

Results: Three articles described some morphological properties and quantitative changes of age related mitoses in TDL in norm. One research was dedicated to quantitative and morphological features of TDL lymphocytes in norm and thyroxintreated. Several studies were focused on physiology of angiotensins, insulin, steroid hormones. A lot of latest publications focus on mediators of proliferation and mitosis. Interleukins are being researched in lymph in antigen-stimulation and other impacts. Age influence on immunity system, on mitoses in particular was revealed. In spite of the progress in the field of drug design there is a view about unsuccessful attempts to solve some problems by reductionist methods. The development of drug design needs to accomplish several complex multistep schemes, control cytotoxicities and then compare the results.

Conclusion: The above mentioned data show the immune system changes with age, in norm and in impact. The changes and difference of TDL mitosis mediators in norm of mature and immature organisms have not been studied. It is necessary to develop new drug components. It is suggested that the changes and difference of TDL mitosis mediators between healthy newborn (neonatal, immature) and adults (mature) can be used to get a key to new drug design for cancer, graft-versus-host disease and other diseases.

Keywords: drug design, age-related mitosis, thoracic duct lymph
Volume 2 Issue 2 - 2018

\author{
Kuznetsov A,' Almabayev Y,' Fackhradiev I, ${ }^{3}$ \\ Almabaeva A, ${ }^{4}$ Serikpayev J, ${ }^{5}$ Tanabayev $B^{6}$ \\ 'Department of Human Anatomy, Novosibirsk State Medical \\ University, Russia \\ ${ }^{2}$ Department of the clinical anatomy, Kazakh National Medical \\ University, Kazakhstan \\ ${ }^{3}$ Laboratory of Experimental Medicine, Kazakh National Medical \\ University, Kazakhstan \\ ${ }^{4}$ Department of the human anatomy with OS, Medical \\ University Astana, Kazakhstan \\ ${ }^{5}$ Human anatomy OS, Medical University Astana, Kazakhstan \\ ${ }^{6}$ Department morphological South Kazakhstan state \\ pharmaceutical academy, Kazakhstan
}

Correspondence: Alexander V Kuznetsov, Department of Human Anatomy, Novosibirsk State Medical University, Russia, Tel+738391378308 76, Email alexapex@yandex.ru

Received: February 15, 2018 | Published: March 05, 2018
Abbreviations: TDL, thoracic duct lymph; DLN, draining lymph nodes

\section{Introduction}

Cell division (mitosis) and gamete production (meiosis) are fundamental requirements for normal organism development. The mammalian cell cycle is tightly regulated by different checkpoints ensuring complete and precise chromosomal segregation and duplication. ${ }^{1,2}$ Agreat amount of basic research over the past few decades has provided unprecedented insights into the highly complex process of cell division. ${ }^{3}$ Mitosis is age-related process..$^{4-6}$ There are mitoses in different normal tissues and they depict the results of mediators effects. So, some studies are focused on the effects of angiotensins, insulin, steroid hormones and kinases..$^{7-13}$ The enormous number of latest publications focus on mediators of proliferation and mitosis. ${ }^{14-16}$ Interleukins of peripheral lymph and TDL in antigen-stimulation and other impacts are being studied. ${ }^{17-22}$ several articles describe some morphological properties and quantitative changes of age-related mitoses of TDL in healthy adults. ${ }^{23-26}$ One research describes the quantity and some morphological features of TDL lymphocytes in norm and thyroxin-treated. Some authors place an emphasis on the development of new drug gable components ${ }^{4}$ and underline that the development of drug design needs to accomplish several complex multistep schemes, control cytotoxicities and then compare the schemes. ${ }^{15,27}$ In spite of the progress in the field of drug design there is a view about unsuccessful attempts (in a latest publication) to solve some problems by reductionist methods and concentration on the parts of the system for which molecular information is available. ${ }^{28}$ Natural compounds from various plants, microorganisms and marine species play an important role in the discovery of new components that can be successfully used in numerous biomedical applications, including anticancer therapeutics. ${ }^{29}$ Our own preliminary (unpublished) data suggest that there can be negative correlation between mitoses number and lymphocytes number in TDL of healthy rabbits in the age range (from immature to mature). Our goal is to assess available researches relevant to age-related mitoses, cytokines and hormones in lymph (body fluid) and, in particular, in TDL of normal newborn (neonatal, immature), adults (mature) and map out a new way to drug design and development. 


\section{Methods}

We investigated the data banks of PubMed/Medline, World Cat, Google and Index Copernicus searching for articles published in the past four decades and valued available researches relevant to the following: 1) age-related mitoses, 2) cytokines and hormones in lymph (body fluid), 3) mitoses, cytokines and hormones in TDL in healthy newborn (neonatal, immature), 4) mitoses, cytokines and hormones in TDL in adults (mature). Four hundred forty five articles have been analyzed. The analyzed data suggest mapping out a new way to drug design and development.

\section{Results}

Three articles described some morphological properties and quantitative changes of age related data about mitoses of TDL in norm. One research was dedicated to quantitative and morphological features of TDL lymphocytes in norm and thyroxin-treated. Several studies were focused on physiology angiotensins, insulin, steroid hormones and kinases. The enormous number of latest publications focus on mediators of proliferation and mitosis. Interleukins are being researched in lymph in antigen-stimulation and other impacts. Age influence on immunity system, mitoses, in particular, was established. In spite of the progress in the field of drug design there is a view about unsuccessful attempts to solve some problems by reductionist methods and concentration on parts of the system for which molecular information is available. The development of drug design needs to accomplish several complex multistep schemes, control cytotoxicities and then compare the results.

\section{Discussion}

Although we have analyzed four hundred forty five articles not all of them have been inserted in the article. Only more appropriate studies for the article aim have been used. The results of the analyses show that cell division (mitosis) and gamete production (meiosis) are fundamental requirements for normal organism development. The mammalian cell cycle is tightly regulated by different checkpoints ensuring complete and precise chromosomal segregation and duplication. A colossal amount of basic research over the past few decades has provided unprecedented insights into the highly complex process of cell division. There is an ever-expanding catalogue of proteins that orchestrate, participate and coordinate the exquisite processes of spindle formation, chromosome dynamics and the formation and regulation of kinetochore-microtubule attachments. The use of classical microtubule poisons has still been widely and often successfully used to combat a variety of cancers, but their nonselective interference in other crucial physiologic processes necessitate the identification of novel druggable components specific to the cell cycle/division pathway. Interleukins of peripheral lymph and DTL in antigen-stimulation and other impacts were studied. ${ }^{30,31}$ Several articles describe some morphological properties and quantitative changes of age-related mitoses of TDL in normal adults. Some authors emphasize the development of new druggable components and claim that the development of drug design needs to accomplish several complex multistep schemes, control cytotoxicities and then compare the schemes. ${ }^{30-32}$ Flow cytometry revealed increasing levels of different suppressive myeloid cells in lymphoid organs: MDSCs dominated bone marrow (BM) and spleens, M2 macrophages dominated tumor-draining lymph nodes (DLN) and a mixed IL-10(+) TNF- $\alpha(+)$ CD206(-) CX3CR1(+) M1/M2 (M3) macrophage subset dominated the mesothelioma microenvironment. Ki67 staining and cell cycle analysis showed that tumor-associated M1 and M3, but not M2, macrophages were proliferating in situ, with M1 cells arrested in the G1 phase while M3 cells progressed to mitosis. It is known that mitosis is age-related process as well as other components of immune system. ${ }^{33-35}$ It was posited that a combination of adjuvantation systems may provide enhanced immune activation but has typically been developed without regard to the age of the target population and distinct combinations of TLRAs and C-type lectin receptor agonists may enhance Th1 responses of newborn DCs. Neutrophils, monocyte/macrophages and dendritic cells, which first recognize and respond to infection, show age-related impairment of many functions relevant to anti-viral responses. Natural killer cells control many viral infections and show age-related changes in phenotype and functional responses. Researchers keep searching of reduced response of aged DCs to RA enhances mucosal inflammation in the elderly, increasing their susceptibility to mucosal diseases. To obtain the mitoses the original method of obtaining central lymph from the cistern chili of thoracic duct was used. TDL sampling was performed with original glass micropipettes (as an injection needle). Mitoses were revealed in TDL of anesthetized intact rabbits "Chinchilla": 50 adult rabbits group 1, 15 immature rabbits - group 2. The specimens of cells were transferred (as smears) to the microscopic slide, stained with Giemsa and studied with a light microscope. Various mitoses stages were revealed in TDL of immature rabbits (16-25 mitoses in every smear). Asymmetric mitoses and the following stages of mitoses: prophase, metaphase, anaphase, telophase, cytokinesis were not revealed in TDL of adult rabbits. The results of the investigation confirm the existence of the mitotic factor in TDL. The revealed quantitative differences of age-related mitoses in TDL open a new approach to the investigation of physiological function and regulation of mitoses. Mitosis has a recurring character. The results of the study suggest a new approach to the creation of cancer vaccines and other drugs. ${ }^{26}$ Similar changes can be revealed in plants. It is described that natural compounds from various plants, microorganisms and marine species play an important role in the discovery of novel components that can be successfully used in numerous biomedical applications, including anticancer therapeutics. ${ }^{29}$ Now researchers keep searching for the combination of adjuvantation systems to provide immune system correction. At the same time, in spite of the progress in the field of drug design there is a view about unsuccessful attempts to solve some problems by reductionist methods and concentration on parts of the system for which molecular information is available. ${ }^{28}$

\section{Conclusion}

The above mentioned data show immune system changes with aging, in norm and in impact. Changes and differences of TDL mitosis mediators in healthy mature and immature organisms have not been studied. It is necessary to develop new drug gable components. The mechanism of the change of age-related mitosis and mediators changes in TDL of immature and mature organisms in norm is unclear. It is suggested that the changes and difference of TDL mitosis mediators between healthy newborns (neonatal, immature) and adults (mature) can be used to get a key to a novel design of new drugs for cancer, graft-versus-host disease and other diseases. Natural age-related changes and difference of mitoses and TDL mediators of healthy immature and mature organisms can be used as a basic predictive mitosis model for the development strategy in wide biological and medical fields. 


\section{Acknowledgement}

We thank Galina Kuznetsova for help in translation.

\section{Conflict of interest}

The authors declare that the research was conducted in the absence of any commercial or financial relations that could be construed as a potential conflict of interest.

\section{References}

1. Cortés-López M, Gruner MR, Cooper DA, et al. Global accumulation of circ RNAs during aging in Caenorhabditis elegans. BMC Genomics. 2018;19(1):2-12.

2. Zhao ZX, Feng XB, Shi T, et al. The comparison of CD4(+) CD25(+) Treg, IL-10 and TGF-beta from lymph and blood in bronchial asthmatic rat and the effect of dexamethasone on it. Xi Bao Yu Fen Zi Mian Yi Xue Za Zhi. 2010;26(3):238-241.

3. Tan EP, Duncan FE, Slawson C. The sweet side of the cell cycle. Biochem Soc Trans. 2017;45(2):313-322.

4. Agarwal S, Varma D. Targeting mitotic pathways for endocrine-related cancer therapeutics. Endocr Relat Cancer. 2017;24(9): T65-T82.

5. Polymenis M, Kennedy BK. Unbalanced Growth, Senescence and Aging. Adv Exp Med Biol. 2017;(1002):189-208.

6. Myles A, Gearhart PJ, Cancro MP. Signals that drive T-bet expression in B cells. Cell Immunol. 2017;(321):3-7.

7. Reddy MK, Baskaran K, Molteni A. Inhibitors of angiotensin-converting enzyme modulate mitosis and gene expression in pancreatic cancer cells. Proc Soc Exp Biol Med. 1995;210(3):221-226.

8. Shirakawa J, Fernandez M, Takatani et al. Insulin Signaling Regulates the FoxM1/PLK1/CENP-A Pathway to Promote Adaptive Pancreatic $\beta$ Cell Proliferation. Cell Metab. 2017;25(4):868-882.

9. Hope JC, Sopp P, Collins RA, et al. Differences in the induction of CD8+ T cell responses by subpopulations of dendritic cells from afferent lymph are related to IL-1 alpha secretion. J Leukoc Biol. 2001;69(2):271-279.

10. Yang J, Harris AL, Davidoff A. Hypoxia and Hormone-Mediated Pathways Converge at the Histone Demethylase KDM4B in Cancer. Int J Mol Sci. 2018;19(1):240.

11. Beltz BS, Benton JL. From Blood to Brain: Adult-Born Neurons in the Crayfish Brain Are the Progeny of Cells Generated by the Immune System. Front Neurosci. 2017;(11):662.

12. Persson WK, Colditz IG, Lun S, et al. Cytokines in mammary lymph and milk during endotoxin-induced bovine mastitis. Res Vet Sci. 2003;74(1):31-6.

13. Marston AL, Wassmann K. Multiple Duties for Spindle Assembly Checkpoint Kinases in Meiosis. Frontiers in Cell and Developmental Biology. 2017;(5):109.

14. Liewer S, Huddleston A. Alisertib: a review of pharmacokinetics, efficacy and toxicity in patients with hematologic malignancies and solid tumors. Expert Opin Investig Drugs. 2018;27(1):105-112.

15. Yin X, Xu X, Zhao Y, et al. Comparison of Several Optimization Schemes for the Induction and Expansion of Antibody-Mediated High Efficiency CIK (AMHE-CIK). In vitro. 2016;24(1):191-196.

16. Bujdoso R, Young P, Hopkins J, et al. Non-random migration of CD4 and CD8 T cells: changes in the CD4: CD8 ratio and interleukin
2 responsiveness of efferent lymph cells following in vivo antigen challenge. Eur J Immunol. 1989;19(10):1779-1784.

17. Olszewski WL, Grzelak I, Ziolkowska A, et al. Epidermal cell thymocyte activity factor/interleukin 1 (ETAF/IL)-like activity in lymph drained from normal human skin. Lymphology. 1988;21(2):118-123.

18. Bujdoso R, Young P, Hopkins J, et al. IL-2-like activity in lymph fluid following in vivo antigen challenge. Immunology. 1990;69(1):45-51.

19. Ernström U, Larsson B. Thymic and thoracic duct contributions to blood lymphocytes in normal and thyroxin-treated guinea-pigs. Acta Physiol Scand. 1966;66(1):189-195.

20. Davidson MT, Deitch EA, Lu Q, et al. A study of the biologic activity of trauma-hemorrhagic shock mesenteric lymph over time and the relative role of cytokines. Surgery. 2004;136(1):32-41.

21. Xiao H, Wang DC, Leng XF, et al. The changes in the tumor necrosis factor alpha, interleukin-6 and interleukin-8 levels in the lymph and of the dynamics of the lymphokines during shock stage of rats with major burns. Chinese journal of burns. 2005;21(2):132-134.

22. Semaeva E, Tenstad O, Skavlan DJ, et al. Access to the spleen microenvironment through lymph shows local cytokine production, increased cell flux, and altered signaling of immune cells during lipopolysaccharide-induced acute inflammation. $J$ Immunol. 2010;184(8):4547-4556.

23. Kuznetsov A, Almabayev Y, Fackhradiev I, et al. The Aging-related Approach to Detection of Mitotic Factor in Thoracic Duct Lymph of Rabbits. International Journal of Innovative Medicine and Health Science. 2017;9:1-4.

24. Kuznetsov A. Dendritic Cells Mitoses in Thoracic Duct Lymph of Immature Rabbits. Transylvanian Review. 2017;(25):3678-3681.

25. Kuznetsov A. Conception of a New Approach to Detection of Lymph Humor Factor in Thoracic Duct Lymph of Rabbits. International Journal of Animal Biology. 2015;(4):110-113.

26. Kuznetsov A. Mitoses in thoracic duct lymph of rabbits. Scientia Agriculture. 2015;9(2):89-92.

27. Lee JJ, Kim KB, Heo J, et al. Protective effect of Arthrospira platensis extracts against ultraviolet B-induced cellular senescence through inhibition of DNA damage and matrix metalloproteinase-1 expression in human dermal fibroblasts. J Photochem Photobiol B. 2017; (173):196203.

28. Van Regenmortel MHV. Development of a Preventive HIV Vaccine Requires Solving Inverse Problems Which Is Unattainable by Rational Vaccine Design. Front Immunol. 2018;8:1-11.

29. Bailon-Moscoso N, Cevallos-Solorzano G, Romero-Benavides JC, et al. Natural Compounds as Modulators of Cell Cycle Arrest: Application for Anticancer Chemotherapies. Curr Genomics. 2017;18(2):106-131.

30. Van Haren SD, Dowling DJ, Foppen W, et al. Age-Specific Adjuvant Synergy: Dual TLR7/8 and Mincle Activation of Human Newborn Dendritic Cells Enables Th1 Polarization. J Immunol. 2016;197(11):4413-4424.

31. Yamashita A, Fukumoto T, Miyamoto M. Studies on lymph humoral factor. Evidence for a lymphocytopoietic factor in rat thoracic duct lymph. Immunology. 1976;30(3):349-59.

32. Jackaman C, Yeoh TL, Acuil ML, et al. Murine mesothelioma induces locally-proliferating $\quad \mathrm{IL}-10(+) \mathrm{TNF}-\boldsymbol{\alpha}(+) \mathrm{CD} 206(-) \mathrm{CX} 3 \mathrm{CR} 1(+) \quad \mathrm{M} 3$ macrophages that can be selectively depleted by chemotherapy or immunotherapy. Oncoimmunology. 2016;5(6): e1173299. 
33. Coiffard B, Pelardy M, Loundou AD, et al. Effect of Immunosuppression on Target Blood Immune Cells Within 1 Year After Lung Transplantation: Influence of Age on T Lymphocytes. Ann Transplant. 2018;(23):11-24.

34. Montgomery RR. Age-related alterations in immune responses to West Nile virus infection. Clin Exp Immunol. 2017;187(1): 26-34.
35. Agrawal S, Ganguly S, Tran A, et al. Retinoic acid treated human dendritic cells induce $T$ regulatory cells via the expression of CD141 and GARP which is impaired with age. Aging (Albany NY). 2016;8(6):12231235. 